\title{
Gains Without Inversion in Quantum Systems with Broken Parities
}

\author{
W. Z. $\mathrm{Jia}^{1, * *}$ and L. F. Wei ${ }^{1, \dagger}$ \\ ${ }^{1}$ Quantum Optoelectronics Laboratory, \\ Southwest Jiaotong University, Chengdu 610031, China
}

(Dated: March 3, 2022)

\begin{abstract}
For a quantum system with broken parity symmetry, selection rules can not hold and cyclic transition structures are generated. With these loop-transitions we discuss how to achieve inversionless gain of the probe field by properly setting the control and auxiliary fields. Possible implementations of our generic proposal with specific physical objects with broken parities, e.g., superconducting circuits and chiral molecules, are also discussed.
\end{abstract}

PACS numbers: 42.50.Gy, 85.25.-j, 42.50.Hz, 32.80.Qk

*Electronic address: jiawenz1979@126.com

†Electronic address: Ifwei@home.swjtu.edu.cn 


\section{INTRODUCTION}

It is well known that under the usual electric-dipole approximation, natural atoms obey the optical selection rules, since their quantum states have well-defined parity symmetries. With these electric-dipole transitions, strong interactions between the fields and atoms have been utilized to dynamically manipulate quantum coherence. As a consequence, many interesting optical phenomena, such as coherent population trapping (CPT) [1], electromagnetically induced transparency (EIT) [2], lasing without inversion (LWI) [3] and so on, can be implemented. Basically, these phenomena are originated from the absorption cancelation via quantum interference of various allowed dipole transitions. Specifically, LWI provides an approach to demonstrate optical gain without requiring population inversion of atomic levels.

Besides the usual electric-dipole transitions, various relatively-weak magnetic-dipole transitions are also utilized to realize certain transitions forbidden by electric-dipole selection rules. Typically, for a natural three-level atom two electric-dipole transitions (between quantum states with different parities, e.g., $|1\rangle$ and $|2\rangle$, and $|2\rangle$ and $|3\rangle$ ) and magnetic-dipole transition (between near degenerate quantum states with same parities, i.e. $|1\rangle$ and $|3\rangle$ ) can generate a loop-transition structure [4-7]. Such loop-transition configurations have been used to control phenomena associated with atomic coherence, including CPT [4], EIT [5], group velocity control [6] and LWI [7].

Recently, certain quantum systems with broken parity symmetries had been investigated. These systems include, e.g., chiral molecules [ $[8-10]$, asymmetric quantum wells [8] , and superconducting quantum circuits (SQCs) [11-14], etc. Both quantum bound states and interaction Hamiltonian (transition matrix elements) in these parity-broken systems have not well defined parities, and thus the usual selection rules do not hold. Therefore, certain particular transition structures, e.g., threelevel $\Delta$-type cyclic transition, can be realized $[8-10,12]$. Compared with loop structure in natural atoms, in parity-broken systems, even three levels are well separated from each other, the possible transition channels can form a loop for selection rules do not hold. Note that such a configuration has been experimentally demonstrated with circuit quantum electrodynamics (QED) systems [13], and has already been utilized to achieve tunable coupling between two flux qubits [14].

In this paper, we investigate how to generate gain without inversion in parity-broken threelevel quantum systems by utilizing the $\Delta$-type transition structure. We consider a $\Delta$-type threelevel system interacting simultaneously with three external fields; a weak probe, a strong coherent 
control, and a tunable auxiliary ones. The optical response of a quantum system with broken-parity symmetry is sensitive to the relative phase of the three coherent driving fields. We show that the desirable inversionless gains (called lasers or masers) of the weak probe field can be achieved by properly controlling the parameters of applied driving fields.

Compared with the previous schemes for realizing LWIs with the loop-transition configurations in microscopic natural atoms [7], we emphasize that: (i)the LWI could also be demonstrated with certain macroscopic quantum systems, such as SQCs; (ii) gain without inversion can be generalized from the traditionally optical waveband (with natural atoms) to the microwave domain (since the energy splittings of SQCs are just in this waveband); and (iii) manipulating the cyclic transitions in the present parity-broken artificial atoms to realize the LWI is relatively simple. This is because one of three transitions in loop configuration with natural atoms is usually implemented by using a significantly-weak magnetic-dipole transition. However, in the loop structure with parity-broken artificial atoms, the strengths of three transitions could be at the same orders.

The paper is organized as: In Sec. II we firstly give an universal analysis on the gain-absorption properties in quantum systems with parity-broken symmetries. Then, in Sec. III, we discuss how to demonstrate our generic proposals with two class specific physical systems, the superconducting flux qubits and the chiral molecules. Conclusions and discussions are given in Sec. IV.

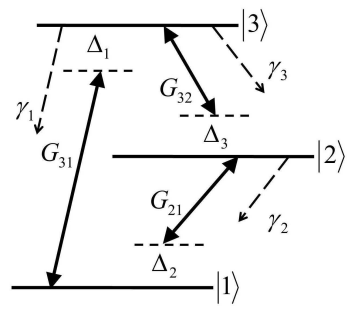

FIG. 1: Three-level quantum system with broken parity. The triangle-shaped transition structure allows three possible transition channels coherently driven by the fields with Rabi frequencies $G_{i j}$ and detunings $\Delta_{i}, i, j=1,2,3 . \gamma_{i}$ are the decay rates of the corresponding levels. 


\section{GAIN WITHOUT POPULATION INVERSION IN QUANTUM SYSTEMS WITH $\triangle$-TYPE CYCLIC TRANSITIONS}

Consider a three-level system with a cyclic transition structure shown in Fig. 1. In our inversionless gain scheme with parity-broken three-level systems, three coherent driving fields are applied; one is the strong resonant-coupling field, one is applied as a probe, and the third one is a tunable auxiliary field. Depending on the specific systems adopted, these three applied fields could be either microwaves or optical waves. The Hamiltonian of our generic system can be written as $H=\sum_{i=1}^{3} E_{i}|i\rangle\langle i|+\frac{1}{2} \sum_{i>j=1}^{3}\left[G_{i j} e^{i \omega_{i j} t}|i\rangle\langle j|+\right.$ H.c. $]$, where $E_{i}$ are the eigenvalues of energy eigenstates $|i\rangle, \omega_{i j}$ and $G_{i j}$ are the frequencies and Rabi frequencies of the coherent driving fields, respectively. Let $\Delta_{1}=E_{3}-E_{1}-\omega_{31}, \Delta_{2}=E_{2}-E_{1}-\omega_{21}$ and $\Delta_{3}=E_{3}-E_{2}-\omega_{32}$ be the detunings of the applied driving fields. When the condition $\Delta_{1}=\Delta_{2}+\Delta_{3}$ is satisfied and in interaction picture, the interaction Hamiltonian is time independent: $H_{I}=\Delta_{1}|3\rangle\left\langle 3\left|+\Delta_{2}\right| 2\right\rangle\langle 2|+\frac{1}{2} \sum_{i>j=1}^{3}\left[G_{i j}|i\rangle\langle j|+\right.$ H.c. $]$. The dynamical evolution of the system, including relaxation terms $\gamma_{i}$, is governed by the Liouvillian equation: $d \rho / d t=-i\left[H_{I}, \rho\right]+\mathcal{L}[\rho]$.

The closed-loop atomic configuration considered here makes the optical properties of system are sensitive to the relative phases of applied fields [5]. The Rabi frequencies $G_{i j}$ should be dealt as

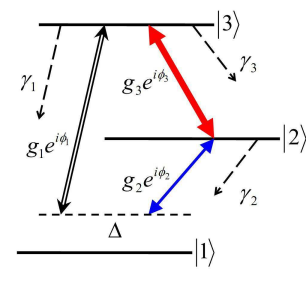

(a)

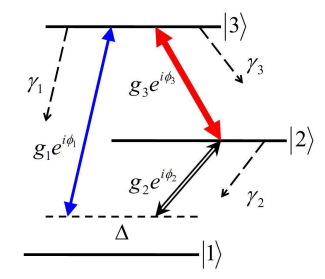

(b)

FIG. 2: (Color online) A $\Delta$-type three-level system driven by three coherent fields: (a) A strong coupling field (red line) applied resonantly to the transition channel $|2\rangle \longleftrightarrow|3\rangle$, a weak probe (blue line) and another auxiliary field ( $\Leftrightarrow$ line) with equal detunings are applied to the transition channels $|1\rangle \longleftrightarrow|2\rangle$ and $|1\rangle \longleftrightarrow|3\rangle$, respectively. (b) A strong coupling field applied resonantly to the transition channel $|1\rangle \longleftrightarrow|2\rangle$, a weak probe and another auxiliary field with equal detunings are applied to the transition channels $|2\rangle \longleftrightarrow|3\rangle$ and $|1\rangle \longleftrightarrow|3\rangle$, respectively. 
complex parameters, e.g., $G_{31} / 2=g_{1} e^{i \phi_{1}}, G_{21} / 2=g_{2} e^{i \phi_{2}}$, and $G_{32} / 2=g_{3} e^{i \phi_{3}}$, with $2 g_{i}$ being the amplitudes and $\phi_{i}$ the phases. Redefining the density matrix elements: $\rho_{i i}=\sigma_{i i}, \rho_{13}=\sigma_{13} e^{-i \phi_{1}}$, $\rho_{23}=\sigma_{23} e^{-i \phi_{3}}, \rho_{12}=\sigma_{12} e^{i\left(\phi_{3}-\phi_{1}\right)}$, and from the above Liouvillian equation, one can obtain their equations of motion:

$$
\begin{aligned}
& \dot{\sigma}_{11}=\gamma_{1} \sigma_{33}+\gamma_{2} \sigma_{22}-\left(i g_{1} \sigma_{13}+i g_{3} \sigma_{12} e^{i \Phi}+\text { H.c. }\right) \\
& \dot{\sigma}_{22}=-\gamma_{2} \sigma_{22}+\gamma_{3} \sigma_{33}+\left(i g_{2} \sigma_{12} e^{i \Phi}-i g_{3} \sigma_{23}+\text { H.c. }\right) \\
& \dot{\sigma}_{12}=\left(-\Gamma_{12}+i \Delta_{2}\right) \sigma_{12}-i g_{3} \sigma_{13}+i g_{1} \sigma_{32}+i g_{2} e^{-i \Phi}\left(\sigma_{22}-\sigma_{11}\right) \\
& \dot{\sigma}_{13}=\left(-\Gamma_{13}+i \Delta_{1}\right) \sigma_{13}+i g_{1}\left(\sigma_{33}-\sigma_{11}\right)+i g_{2} \sigma_{23} e^{-i \Phi}-i g_{3} \sigma_{12}, \\
& \dot{\sigma}_{23}=\left(-\Gamma_{23}+i \Delta_{3}\right) \sigma_{23}+i g_{2} \sigma_{13} e^{i \Phi}-i g_{1} \sigma_{21}+i g_{3}\left(\sigma_{33}-\sigma_{22}\right)
\end{aligned}
$$

where $\Phi=\left(\phi_{2}+\phi_{3}-\phi_{1}\right)$ is the relative phase of the applied fields, and $\Gamma_{12}=\gamma_{2} / 2, \Gamma_{13}=$ $\left(\gamma_{1}+\gamma_{3}\right) / 2, \Gamma_{23}=\left(\gamma_{1}+\gamma_{2}+\gamma_{3}\right) / 2$.

For simplicity, we assume that all the decay rates of the levels are equal, namely $\gamma_{i}=\gamma$. The steady-state solution of the master equation can be attained by setting $\dot{\sigma}_{i j}=0$. We first consider the case shown in Fig. 2(a). A resonant coupling field $g_{3}$ is applied to the transition between the intermediate state $|2\rangle$ and the upper state $|3\rangle$, a weak coherent field $g_{2}$ with the detuning $\Delta$ acted as a probe is applied to the transition between the ground state $|1\rangle$ and intermediate state $|2\rangle$, and an auxiliary field $g_{1}$ with the same detuning $\Delta$ couples the levels $|1\rangle$ and $|3\rangle$, respectively. The absorption behavior for the probe $g_{2}$ can be described by $\operatorname{Im}\left(\sigma_{21} e^{-i \Phi}\right)$. For the configuration displayed in Fig. 2(a), it is seen, from Figs. 3(a) and (b), that remarkable gains, i.e., $\operatorname{Im}\left(\sigma_{21} e^{-i \Phi}\right)<$ 0 , can be established, if the modulus of Rabi frequency $g_{1}$ and the relative phase $\Phi$ are modulated appropriately. In fact, the modulation of $\Phi$ can be achieved by fixing $\phi_{2}, \phi_{3}$ and changing the phase of the auxiliary field $\phi_{1}$ only. Typically, when $g_{1}=0.74 \gamma$ and $\Phi=0$ (or $\pi$ ), it is shown clearly that gain dip appears at $\Delta_{2} \approx-9.98 \gamma\left(\right.$ or $9.98 \gamma$ ); when $\Phi=\pi / 2, g_{1}=1.70 \gamma$, two gain regions locate respectively at about $\Delta_{2}<-10 \gamma$ and $\Delta_{2}>10 \gamma$, with the maximum gain points appearing at $\Delta_{2} \approx \pm 12.12 \gamma$; and when $\Phi=3 \pi / 2, g_{1}=6.13 \gamma$, a remarkable probe gain can be established approximately in a wide spectral range from about $-10 \gamma$ to $10 \gamma$, with the maximum gain point being located at $\Delta_{2}=0$. Fig. 3 (d) shows that the Rabi frequencies of the auxiliary fields, used in Figs. 3(a) and 3(b), are optimal for implementing the desirably maximum gains. More interestingly, Fig. 3(c) shows that population inversion $\sigma_{22}-\sigma_{11}$ is always less than zero for any detuning. This indicates that the phase-dependant gains attained in Fig. 3(a) and (b) are inversionless. 

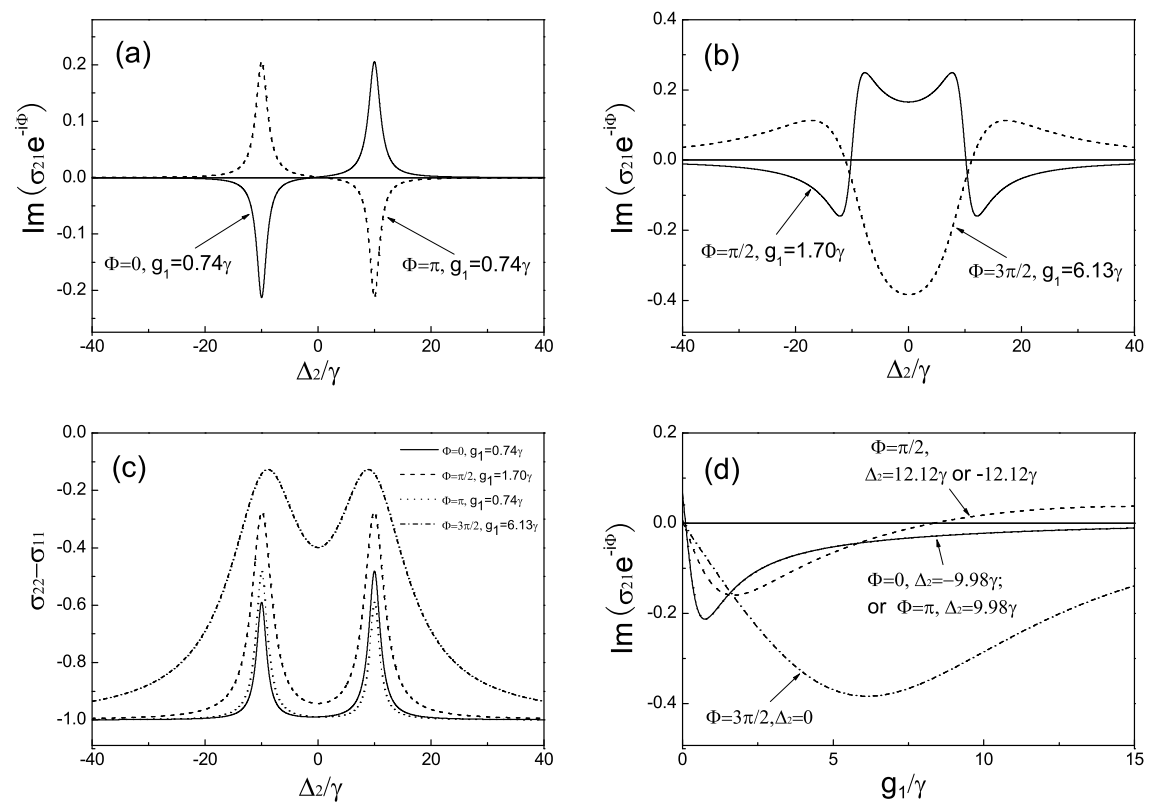

FIG. 3: Phase sensitive probe gain without population inversion of the configuration shown in Fig. 2(a), with the parameters $\gamma_{i}=\gamma, g_{3}=10 \gamma$ and $g_{2}=0.1 \gamma$ : (a) Detuning-dependent probe gain for $g_{1}=0.74 \gamma$ and $\Phi=0$ (solid line); $\pi$ (dashed line). (b) Detuning-dependent probe gain for $\Phi=\pi / 2, g_{1}=1.70 \gamma$ (solid line) and $\Phi=3 \pi / 2, g_{1}=6.13 \gamma$ (dashed line). (c) Detuning-dependent population difference $\sigma_{22}-\sigma_{11}$ corresponds to the probe gains shown in (a) and (b). It is clearly shown that these gains are not due to the population inversions. (d) Probe gain versus amplitude of Rabi frequency of auxiliary field $g_{1}$. With fixed relative phases and detunings, maximal gains can be gotten at $g_{1}=0.74 \gamma$ (solid line); $1.70 \gamma$ (dashed line); or $6.13 \gamma$ (dash-dotted line). This means that the parameters of auxiliary field utilized in (a) and (b) for getting the gains are optimal.

On the other hand, if the atomic configuration in Fig. 2(b) is selected, i.e., $g_{2}$ acts as a resonant coupling field, $g_{3}$ is a weak probe with detuning $\Delta$, and $g_{1}$ is an auxiliary field with the same detuning, Figs. 4(a-c) show similarly that the phase-sensitive gain without population inversion could still be achieved. Specifically, when $g_{1}=0.94 \gamma$ and $\Phi=0$ (or $\pi$ ), it is shown clearly that gain dip appears at $\Delta_{3} \approx 10.04 \gamma\left(\right.$ or $-10.04 \gamma$ ); when $\Phi=\pi / 2, g_{1}=6.97 \gamma$, a remarkable probe gain can be established in a spectral range from about $-20 \gamma$ to $20 \gamma$, with the maximum gain point being located at $\Delta_{3}=0$; and when $\Phi=3 \pi / 2, g_{1}=1.52 \gamma$, two gain regions locate respectively at about $\Delta_{3}<-10 \gamma$ and $\Delta_{3}>10 \gamma$, with the maximum gain points appearing at $\Delta_{3} \approx \pm 12.92 \gamma$. 

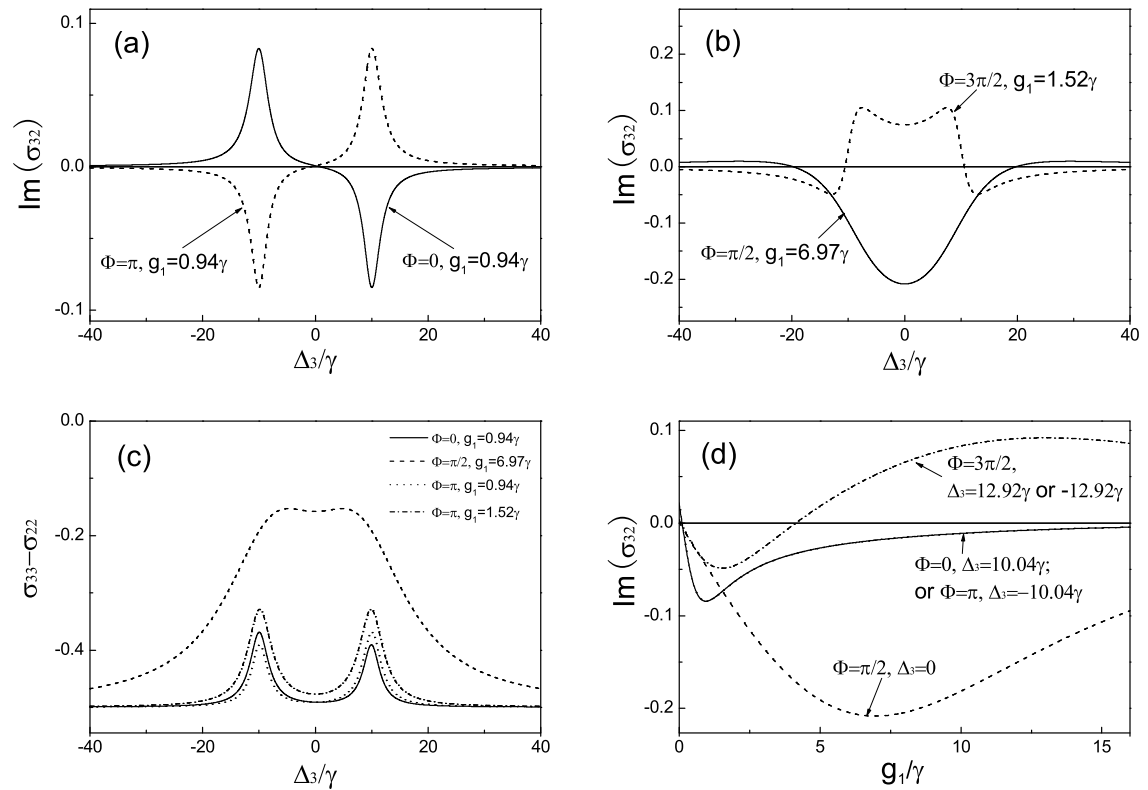

FIG. 4: Phase sensitive probe gain without population inversion of the configuration shown in Fig. 2(b), with the parameters $\gamma_{i}=\gamma, g_{2}=10 \gamma$ and $g_{3}=0.1 \gamma$ : (a) Detuning-dependent probe gain for $g_{1}=0.94 \gamma$ and $\Phi=0$ (solid line); $\pi$ (dashed line). (b) Detuning-dependent probe gain for $\Phi=\pi / 2, g_{1}=6.97 \gamma$ (solid line) and $\Phi=3 \pi / 2, g_{1}=1.52 \gamma$ (dashed line). (c) Detuning-dependent population difference $\sigma_{33}-\sigma_{22}$ corresponds to the probe gains shown in (a) and (b). It is clearly shown that these gains are not due to the population inversions. (d) Probe gain versus amplitude of Rabi frequency of auxiliary field $g_{1}$. With fixed relative phases and detunings, maximal gains can be gotten at $g_{1}=0.94 \gamma$ (solid line); $6.97 \gamma$ (dashed line); or $1.52 \gamma$ (dash-dotted line). This means that the parameters of auxiliary field utilized in (a) and (b) for getting the gains are optimal.

Also, for the fixed $\Phi$ and detuning $\Delta_{3}$, Fig. 4(d) shows how the $\operatorname{Im}\left(\sigma_{32}\right)$ depends on the parameter $g_{1}$. One can see that the parameters selected in Figs. 4(a-c) are optimal for realizing the gains.

The above numerical results can be simply explained by investigating the steady-state condition in Eqs. (3) and (5):

$$
\begin{aligned}
& \operatorname{Im}\left(\sigma_{21} e^{-i \Phi}\right)=g_{2} \Gamma_{12}\left(\sigma_{11}-\sigma_{22}\right) / \mathcal{A}+\operatorname{Im}\left[\frac{1}{\mathcal{A}}\left(\Gamma_{12}-i \Delta_{2}\right)\left(i g_{3} \sigma_{31}-i g_{1} \sigma_{23}\right) e^{-i \Phi}\right] \\
& \operatorname{Im} \sigma_{32}=g_{3} \Gamma_{23}\left(\sigma_{22}-\sigma_{33}\right) / \mathcal{B}+\operatorname{Im}\left[\left(\Gamma_{23}-i \Delta_{3}\right)\left(i g_{1} \sigma_{12}-i g_{2} \sigma_{31} e^{-i \Phi}\right) / \mathcal{B}\right]
\end{aligned}
$$

with $\mathcal{A}=\Gamma_{12}^{2}+\Delta_{2}^{2}, \mathcal{B}=\Gamma_{23}^{2}+\Delta_{3}^{2}$. Clearly, in the configuration shown in Fig. 2(a), in order 
to achieve gain without inversion for probe $g_{2}$ the conditions $\operatorname{Im}\left(\sigma_{21} e^{-i \Phi}\right)<0$, and $\sigma_{11}-\sigma_{22}>$ 0 should be simultaneously satisfied. It can be seen from Eq. (6) that, when $\sigma_{11}-\sigma_{22}>0$, the first term on the right-hand side of Eq. (6) is positive. Thus, in order to get a gain, namely $\operatorname{Im}\left(\sigma_{21} e^{-i \Phi}\right)<0$, the second term on the right-hand side of Eq. (6) must contribute negatively to $\operatorname{Im}\left(\sigma_{21} e^{-i \Phi}\right)$. This implies that in this case the induced inversionless gain is originated from the dynamically induced coherence by the coupling field $g_{3}$ and the auxiliary one $g_{1}$. Clearly, if the auxiliary field is not applied, the system shown in Fig. 2(a) is reduced to the usual ladder-type configuration. In the presence of a control field $g_{3}$ and a probe field $g_{2}$, the phenomenon of EIT can be achieved instead of inversionless gain. However, in the present systems with $\Delta$-type cyclic transition structure (due to the broken parity symmetries), an auxiliary coherent driving field $g_{1}$ could be applied to couple the levels $|1\rangle$ and $|3\rangle$. As a consequence, the term related to $\sigma_{23}$ appears and thus negative values of $\operatorname{Im}\left(\sigma_{21} e^{-i \Phi}\right)$ can be induced within certain spectral ranges. This is clearly proven that the auxiliary driving field plays crucial roles for the appearance of the gain. Similarly, Eq. (7) shows that for the configuration displayed in Fig. 2(b), an auxiliary field $g_{1}$ is necessary to obtain the phase-dependant gain without inversion of the probe field $g_{3}$.

\section{PHYSICAL DEMONSTRATIONS AND POSSIBLE APPLICATIONS}

The above generic results can be realized with all the systems whose quantum states possess broken parity symmetries, such as chiral molecules [8-10], asymmetric quantum wells [8], superconducting quantum circuits (SQCs) [11-14], and so on.

Typically, SQCs can be regarded as artificial atoms with quantized energy levels. Quantummechanical behaviors in these artificial atoms, such as spectroscopy [15], Rabi oscillations [16], and so forth, have already been demonstrated experimentally. Also, SQCs coupling to various bosonic modes (e.g., microwave fields, nano-mechanical resonator [17], superconducting transmission line [18], etc.) can be utilized to simulate various quantum optical phenomena in the microwave domain. Moreover, recent studies show that quantum optical phenomena related to atomic coherence, such as EIT [19, 20], Autler-Townes effects [21] and CPT [22], can also be achieved in SQCs. Here, by using their special loop transition-structure we show that these devices could be utilized to realize another important phenomenon related to atomic coherence, i.e., the microwave gain without population inversion mentioned above.

In SQCs the desirable cyclic transition configurations could be demonstrated with both flux 
and phase qubits [11-14]. In fact, the parity-broken and consequently loop-transition configurations had been first observed in phase-qubit experiments [11] and recently demonstrated with flux qubits [13, 14]. Here, we take the flux qubits as typical examples. For the artificial atoms generated by three Josephson-junction circuits selection rules do not always exist, as the parity of the system can be broken by adjusting the parameters of circuits. The effective potential of the system reads (See, e.g., Refs. [12, 19].) $U\left(\varphi_{m}, \varphi_{p}\right)=2 E_{J}\left(1-\cos \varphi_{p} \cos \varphi_{m}\right)+\alpha E_{J}\left[1-\cos \left(2 \pi f+2 \varphi_{m}\right)\right]$, with $\varphi_{p, m}=\left(\varphi_{1} \pm \varphi_{2}\right) / 2$ being the generalized coordinates defined by the phase drops $\varphi_{1}$ and $\varphi_{2}$ across the two larger junctions, respectively, and $E_{J}$ their Josephson energies. The reduced magnetic flux $f=\Phi_{e} / \Phi_{0}$ is defined as the ratio of the external magnetic flux $\Phi_{e}$ with the flux quantum $\Phi_{0}$, and $0<\alpha<1$. Clearly, if the flux is biased away from the degenerate point with $f=1 / 2$, the potential $U\left(\varphi_{m}, \varphi_{p}\right)$ has ill-defined parities and thus microwave-induced transitions between arbitrary two levels are possible. Typically, it is seen from Ref. [12] that, for $f=0.496$ the lowest three energy levels of the artificial atom are well separated from other higher energy levels and the moduli $\left|t_{i j}\right|$ of transition matrix elements between any two levers are comparable (i.e., $\left|t_{01}\right| \simeq 0.19,\left|t_{02}\right| \simeq 0.14$, and $\left|t_{12}\right| \simeq 0.19$ ). This indicates that the flux qubit is really an ideal candidate to realize the phase-sensitive inversionless gain proposed in Sec. II.

The time scale to reach the steady-state solutions (see, Sec. II) required for realizing LWI with the present SQCs could be estimated as follows. With the experimentally-demonstrated relaxation rate $6.9 \times 10^{7} s^{-1}$ (between the two lower levels of a flux qubit at degenerate point) [20, 23], and the calculated transition matrix elements $\left|t_{i j}(f)\right|$ [12], the three decay rates used in our proposal are estimated as: $\gamma_{2}=\gamma_{3}=5.5 \times 10^{6} \mathrm{~s}^{-1}$, and $\gamma_{3}=3.2 \times 10^{6} \mathrm{~s}^{-1}$ (Note that the relaxation times are proportional to $\left|t_{i j}\right|^{2}$ ). If the Rabi frequencies of coupling and probe fields are setting as $10 \gamma_{2}$ and $0.1 \gamma_{2}$, and by appropriately choosing Rabi frequency of auxiliary field, the time scale to reach the so-called stationary solutions is numerically as $10^{-6} \mathrm{~s}$. This implies that the physical demonstration of our proposal with SQCs should be feasible.

Furthermore, constructing an effective medium to realize inversionless maser with these artificial atoms is also possible. In fact, the typical size of a superconducting qubit is about $10^{-6} \mathrm{~m} \mathrm{[16].}$ This is much smaller than the wavelength of microwave. For example, if a transition can be driven by microwave field with wavelength around $10^{-2} \mathrm{~m}$, the ratio between the wavelength of driving field and the size of artificial atoms is about $10^{4}$. This is in accordance with the ratio between the optical wavelength and the size of natural atom. Therefore, an effective medium generated by a block consisting of superconducting artificial atoms is feasible. 
For another kind quantum system with broken parity symmetry, i.e., chiral molecules [8-10], our scheme proposed in Sec. II, could also offer an effective way to discriminate the left- and righthanded chiral molecules (Such pairs are called "enantiomers" [8].), in addition to realize LWI. If only the three lowest levels are considered, a chiral molecule can be modeled as a three-level cyclic system as shown in Fig. 1. Thus three lasers can be applied to enantiomeric molecules with the Rabi frequencies being chosen as, for example, those in Fig. 3(a). The Rabi frequencies of applied lasers between any pair of left- and right-handed states differ by a sign, namely, $g_{i} e^{i \phi_{i}^{L}}=-g_{i} e^{i \phi_{i}^{R}}$ $(i=1,2,3)$. Thus the according phase factors of Rabi frequencies $\phi_{i}^{L, R}(i=1,2,3)$ differ by $\pi$ [8]. Clearly, the difference between the total phase factors of the two enantiomers is $\Phi^{L}-\Phi^{R}=\pi$, where $\Phi^{L, R}=\phi_{2}^{L, R}+\phi_{3}^{L, R}-\phi_{1}^{L, R}$. On the other hand, as shown in Sec. II, the gain-absorption properties of the two enantiomers (both with cyclic transition structures) are dependent on the total phase factors $\Phi^{L, R}$. Clearly, if we set the phase factor of the applied coherent fields appropriately to assure that $\Phi^{L}=\pi$, then inevitably $\Phi^{R}=0$. As a consequence, the probe gain-absorption spectra of the left- and right-handed chiral molecules correspond to the dashed and solid lines in Fig. 3(a), respectively. Thus the enantiomers can be identified by their different gain-absorption spectra.

\section{CONCLUSIONS AND DISCUSSIONS}

In summary, we have shown that phase-sensitive gain without inversion can be realized with parity-broken quantum systems. We investigate two typical inversionless gain approaches by applying a probe, a coupling field, and a tunable auxiliary field to generate a transition loop. In these approaches, by modifying the phase and modulus of Rabi frequency of auxiliary field, remarkable inversionless gains can be obtained for different probe detunings. Our generic proposal could be implemented with various specific systems with broken parity symmetries, e.g., superconducting artificial atoms, chiral molecules, asymmetric quantum wells and so on. Therefore, maser (laser) without inversion can be realized in principle with these systems by using their cyclic transition structures. As far as chiral molecules, the phase-dependent gain-absorption spectra may be used to discriminate enantiomeric molecules. 


\section{Acknowledgments}

The project was supported in part by National Natural Science Foundation of China under

Grant Nos. 10874142, 90921010, and the National Fundamental Research Program of China through Grant No. 2010CB923104.

[1] E. Arimondo, in Progress in Optics XXXV, edited by E. Wolf (North-Holland, Amsterdam, 1996).

[2] S. E. Harris, J. E. Field, and A. Imamoglu, Phys. Rev. Lett. 641107 (1990); K. J. Boller, A. Imamoglu, and S. E. Harris, Phys. Rev. Lett. 66, 2593 (1991); S. E. Harris, Phys. Today 50, 36 (1997); M. Fleischhauer, A. Imamoglu, and J. P. Marangos, Rev. Mod. Phys. 77, 633 (2005).

[3] S. E. Harris, Phys. Rev. Lett. 62 (1989) 1033; M. O. Scully, S. Y. Zhu, and A. Gavrielides, Phys. Rev. Lett. 62, 2813 (1989); A. Imamoglu, J. E. Field, S. E. Harris, Phys. Rev. Lett. 661154 (1991); E. S. Fry, X. Li, D. Nikonov, G. G. Padmabandu, M. O. Scully, A. V. Smith, F. K. Tittel, C. Wang, S. R. Wilkinson, and S. Y. Zhu, Phys. Rev. Lett. 70, 3235 (1993); A. S. Zibrov, M. D. Lukin, D. E. Nikonov, L. Hollberg, M. O. Scully, V. L. Velichansky, and H. G. Robinson, Phys. Rev. Lett. 75, 1499 (1995); J. Mompart and R. Corbalan, J. Opt. B: Quantum Semiclass. Opt. 2, R7 (2000).

[4] D. V. Kosachiov, B. G. Matisov, and Yu. V. Rozhdestvensky, Opt. Commun. 85, 209 1991; D. V. Kosachiov, B. G. Matisov and Yu. V. Rozhdestvensky, J. Phys. B 25, 2473 (1992).

[5] B. P. Hou, S. J. Wang, W. L. Yu and W. L. Sun, J. Phys. B, 38 1419(2005); E. A. Wilson, N. B. Manson, C. Wei and L. J. Yang, Phys. Rev. A 72, 063813, (2005); Hebin Li, V. A. Sautenkov, Y. V. Rostovtsev, G. R. Welch, P. R. Hemmer, and M. O. Scully, Phys. Rev. A 80023820 (2009).

[6] D. Bortman-Arbiv, A. D. Wilson-Gordon, and H. Friedmann, Phys. Rev. A 63043818 (2001).

[7] O. Kocharovskaya, P. Mandel, and Y. V. Radeonychev, Phys. Rev. A 451997 (1992); W. H. Xu, J. H. Wu and J. Y. Gao, Opt. Commun. 223367 (2003).

[8] P. Kra'l and M. Shapiro, Phys. Rev. Lett. 87, 183002 (2001).

[9] P. Kra'l, I. Thanopulos, M. Shapiro, and D. Cohen, Phys. Rev. Lett. 90, 033001 (2003).

[10] Yong Li, C. Bruder, and C. P. Sun, Phys. Rev. Lett. 99, 130403 (2007).

[11] J. M. Martinis, M. H. Devoret and J. Clarke, Phys. Rev. B 354682 (1987); J. Clarke, A. N. Cleland, M. H. Devoret, D. Esteve, and J. M. Martinis, Science 239, 992 (1988).

[12] Yu-xi Liu, J. Q. You, L. F. Wei, C. P. Sun, and F. Nori, Phys. Rev. Lett. 95, 087001 (2005). 
[13] F. Deppe, M. Mariantoni, E. P. Menzel, A. Marx, S. Saito, K. Kakuyanagi, H. Tanaka, T. Meno, K. Semba, H. Takayanagi, E. Solano, and R. Gross, Nat. Phys. 4, 686 (2008).

[14] K. Harrabi, F. Yoshihara, A. O. Niskanen, Y. Nakamura, and J. S. Tsai, Phys. Rev. B 79, 020507(R) (2009).

[15] J. R. Friedman, V. Patel, W. Chen, S. K. Tolpygo, and J. E. Lukens, Nature (London) 406, 43 (2000); C. H. van der Wal, A. C. J. ter Haar, F. K. Wilhelm, R. N. Schouten, C. J. P. M. Harmans, T. P. Orlando, S. Lloyd, and J. E. Mooij, Science 290, 773 (2000); A. J. Berkley, H. Xu, R. C. Ramos, M. A. Gubrud, F. W. Strauch, P. R. Johnson, J. R. Anderson, A. J. Dragt, C. J. Lobb, and F. C. Wellstood, Science 300, 1548 (2003); H. Xu, F. W. Strauch, S. K. Dutta, P. R. Johnson, R. C. Ramos, A. J. Berkley, H. Paik, J. R. Anderson, A. J. Dragt, C. J. Lobb, and F. C. Wellstood, Phys. Rev. Lett. 94, 027003 (2005).

[16] D. Vion, A. Aassime, A. Cottet, P. Joyez, H. Pothier, C. Urbina, D. Esteve, and M. H. Devoret, Science 296, 886 (2002); Chiorescu, Y. Nakamura, C. J. P. M. Harmans, and J. E. Mooij, Science 299, 1869 (2003).

[17] A. N. Cleland and M. R. Geller, Phys. Rev. Lett. 93, 070501 (2004).

[18] A. Wallraff, D. I. Schuster, A. Blais, L. Frunzio, R. S. Huang, J. Majer, S. Kumar, S. M. Girvin, and R. J. Schoelkopf, Nature (London) 431, 162 (2004).

[19] K. V. R. M. Murali, Z. Dutton, W. D. Oliver, D. S. Crankshaw, and T. P. Orlando, Phys. Rev. Lett. 93, 087003 (2004); Z. Dutton, K. V. R. M. Murali, W. D. Oliver, and T. P. Orlando, Phys. Rev. B 73, 104516 (2006).

[20] A. A. Abdumalikov Jr., O. Astafiev, A. M. Zagoskin, Yu. A. Pashkin, Y. Nakamura, and J. S. Tsai, Phys. Rev. Lett. 104, 193601 (2010).

[21] M. Baur, S. Filipp, R. Bianchetti, J. M. Fink, M. Goppl, L. Steffen, P. J. Leek, A. Blais, and A. Wallraff, Phys. Rev. Lett. 102, 243602 (2009); M. A. Sillanpa, Jian Li, K. Cicak, F. Altomare, J. I. Park, R. W. Simmonds, G. S. Paraoanu, and P. J. Hakonen, Phys. Rev. Lett. 103, 193601 (2009).

[22] W. R. Kelly, Z. Dutton, J. Schlafer, B. Mookerji, T. A. Ohki, J. S. Kline and D. P. Pappas, Phys. Rev. Lett. 104, 163601 (2010).

[23] O. Astafiev, A. M. Zagoskin, A. A. Abdumalikov Jr., Y. A. Pashkin, T. Yamamoto, K. Inomata, Y. Nakamura and J. S. Tsai, Science 327, 840 (2010). 